\title{
Damage initialization techniques for non-sequential FE propagation analysis of delaminations in composite aerospace structures
}

\author{
E. Panettieri · D. Fanteria · A. Firrincieli
}

Received: 15 January 2015 / Accepted: 20 May 2015

\begin{abstract}
The experimental effort required to develop, damage tolerant, aerospace composite structures could be significantly reduced if reliable numerical simulations were used to perform engineering studies of complex damaged structures.

Finite Element (FE) simulations of impact damaged structures typically follow a sequential approach that require large computational resources to reproduce complex damage scenarios. A numerical tool capable to reconstruct such scenarios using data from previous impact simulations or NDI could noticeably improve the simulation workflow for damaged composite structures. The paper proposes a method to inizialize the damage variables in numerical analyses aimed at assessing damage propagation, and that are potentially able to evaluate the residual strength of damaged structures. The approach is developed within FE software ABAQUS, and uses SDVINI subroutine to initialize damage variables defined by a user-material-subroutine (UMAT), that provides the constitutive models of the lamina and of the interlaminar layers.
\end{abstract}

\section{E. Panettieri}

University of Pisa, Department of Civil and Industrial Engineering - Aerospace division, via G. Caruso 8, 56122 Pisa, Italy

Tel.: +39-050-2217274

E-mail: enrico.panettieri@for.unipi.it

D. Fanteria

University of Pisa, Department of Civil and Industrial Engineering - Aerospace division, via G. Caruso 8, 56122 Pisa, Italy

Tel.: +39-050-2217266

E-mail: daniele.fanteria@unipi.it

A. Firrincieli

University of Pisa, Department of Civil and Industrial Engineering - Aerospace division, via G. Caruso 8, 56122 Pisa, Italy
Albeit the proposed technique might deal with both inter-laminar and intra-laminar damage, the paper is focused on delaminations.

A user defined traction-separation law is coded in an UMAT that endows ABAQUS cohesive elements with damage initialization capabilities. Then, results of test cases, of increasing complexity, are presented in order to assess the damage initialization procedure and verify the performances of its different operating modes. Two test-cases are based on plate-like specimens for which literature data exist: the first is relevant to a circular artificial delamination while the second presents multiple delaminations caused by an impact and measured via NDI techniques. The last test-case is a stiffened panel which incorporates the typical complexities of aerospace structures, but is still tractable with the sequential simulation approach whose results are used as a term of comparison.

Keywords Composites impact damage - Composites delamination - Damage propagation analyses · Finite Element

\section{Introduction}

The response to impacts of aerospace composite structures and the consequent reduction of their strength are typically evaluated by means of long and expensive experimental campaigns [1]. An extensive use of reliable numerical simulations in engineering studies can improve the design of experiments to validate structural solutions and contribute to the reduction of the experimental effort to develop complex, damage tolerant, composite structures [2].

For a composite structure, the numerical simulation of damage caused by impacts, the analysis of the behavior 
of such a damaged structure until failure and, eventually, the prediction of the residual strength is a challenging task. It can be undertaken by endowing FE codes with appropriate elements and material models that can predict both progressive lamina damage and the onset and propagation of delaminations. For the past decade many researchers have been using continuum damage mechanics (CDM) to develop constitutive relationships, that, within $\mathrm{FE}$ analyses, could predict ply damage initiation and propagation [3-10]. Within the same analyses, delaminations have been simulated by means of interface elements formulated according to the Cohesive Zone Model (CZM) [11-15]. Due to their capability to represent mechanisms that control damage development and failure, cohesive elements are widely used to simulate delaminations (see [16] and references therein). The combined modeling approach based on CDM and CZM can be used to perform analyses on damaged structures in order to predict damage propagation and, possibly, to evaluate residual strength. Standard FE simulations of impact damaged structures typically follow a sequential approach: damage is reproduced by simulating the impact event that caused it, then appropriate measures are taken to let the vibrations fade and, finally, the external loading is applied while damage growth is monitored until failure that typically occur when damage extension causes a complete loss of the load bearing capacity of the structure. Therefore, complex damage scenarios can be only created by successively simulating multiple impact events (allowing damping of vibrations between each of them) before performing any analysis on the damaged structure. The resulting set of sequential analyses is time consuming and requires large computational resources. Consequently, an efficient procedure that reconstructs damage scenarios, using results from previous impact simulations or NDI inspections data, would constitute a major improvement for the analysis workflow of complex damaged structures. This paper contributes to such endeavor by proposing a procedure that inizializes the damage variables in numerical analyses of damaged components. Necessarily, a procedure of this type is profoundly linked to the FE software used so, also due to previous experience $[10,17]$, FE software SIMULIA ABAQUS [18], complemented with User MATerial FORTRAN subroutines (UMAT), was selected for this work. The key idea behind the procedure proposed in the paper is to use ABAQUS subroutine SDVINI to initialize every State Damage Variable (SDV) defined by the UMAT subroutines that reproduce the progressive damage of composite laminates. This strategy can be pursued if a user defined traction-separation law is defined, via UMAT, also for the cohesive elements since
ABAQUS built-in cohesive elements cannot be inizialized. This, that apparently seems a drawback, is, instead, an advantage since the definition of dedicated traction separation laws permits to use state-of-the art mixed-mode formulations $[12,13,15,19]$ and to overcome certain known limitations of ABAQUS cohesive elements [20], especially when used in evolving, mixedmode conditions. With such added functionality, the proposed technique can be used to initialize both ply damage and delaminations since they are treated similarly in all the simulations involved. Nonetheless, in this work, in order to reduce the computational effort, especially for the larger models, the intra-laminar damage is momentarily deactivated and only delamination phenomena are simulated. The initialization procedure can operate in different modes depending on the complexity of the delamination scenario that one wants to create. Simple delamination shapes (i.e. circular or rectangular), like those of artificial defects obtained in practice my means of teflon inserts between the plies, may be defined analytically within the SDVINI subroutine. Conversely, the geometric definition of multiple delaminations with arbitrary shapes, like those that can be reconstructed from NDI ultrasonic scanning images, has to be loaded by the SDVINI from external files. Finally, in the most general case, a complete of damage transfer between different simulations is obtained by means of two sets of dedicated external scripts that interacts with the SDVINI. In this case, the first set extracts and stores damage data from results database files of the first analysis while the second one loads and re-organizes damage information and passes it to the SDVINI that initializes damage variables of the second analysis. ABAQUS Standard (Dynamic Implicit solver) analyses were performed on test cases, of increasing complexity, in order to assess the proposed procedure for damage initialization and verify the performances of the different operating modes. The first case is relevant to an artificially damaged CAI specimen for which literature data exist about delamination buckling with stable damage growth [21]. In the second case, an impact damage scenario on a CAI specimen is reconstructed from literature information [22] and damage growth during compression is simulated numerically. The last test-case is relevant to a stiffened panel which incorporates the typical complexities of aerospace structures, but is still tractable with the standard sequential simulation approach. The results of stable damage growth during indentation of such sequential simulation are used as a term of comparison for the analysis of the same component with damage initialized by means of the proposed procedure. The remainder of the paper is organized as follows. In the next section, the key as- 
pects of the mixed-mode delamination model that is implemented in the UMAT are presented. Section 3 outlines the proposed approach to damage initialization and briefly presents specifics about the different operating modes depending on shape and source of the relevant damage information. Section 4 presents and discusses the results obtained by employing the proposed approach on the selected test-cases. Finally, essential aspects of the proposed approach and the main achievements of the paper are summarized in Sec. 5 .

\section{Inter-laminar damage modeling and simulation}

Interlaminar damage phenomena in advanced composite materials may be modeled by defining cohesive interactions which can approximate the progressive creation of new fracture surfaces. According to the original formulation of Dugdale [23] and Barenblatt [24], CZM hypothesizes a process (softening) zone located ahead of a crack tip where cohesive interactions, or tractions, are related to the interfacial separation, or displacement jump. A cohesive zone model can be combined with FE methods to develop interfacial cohesive elements. Cohesive elements, placed between composite material layers, can effectively simulate delamination processes in standard FE analyses. Such excellent performances are mainly due to the two key constituents of the cohesive zone model, upon which cohesive elements are based: the delamination surfaces kinematics, encompassing strong discontinuities in the displacement field, and the cohesive constitutive relationship which can model variable-mode loading at the delamination front.

One of the major advantages of cohesive elements over other techniques for modeling delaminations in composites is their capability to effectively capture the inception of new delamination fronts (onset) in a pristine laminate. Conversely, existing delaminations are usually simulated by removing cohesive elements, thus leaving cohesive layers ahead of each delamination front where a propagation is expected. Although effective, cohesive element removal has several drawbacks, namely: the technique is very difficult to be automated, intermediate damage levels, especially at delaminations fronts, can not be represented, and finally, the absence of the cohesive layer may allow the inter-penetration of the sub-laminates (compressive stiffness, which is present also when cohesive elements are damaged, is lost). Interpenetration can be prevented by specifying suitable contact conditions between facing sub-laminates in the delaminated areas where cohesive elements have been removed. Element removal and its related problems might be avoided if internal damage parameters of cohesive elements could be inizialized.

Unfortunately, although major FE software packages provide standard 2D and $3 \mathrm{D}$ cohesive elements with various constitutive options, user access to constitutive parameters is limited and damage initialization of standard cohesive elements is not possible. Consequently, we decided to exploit the possibility to couple an ABAQUS 3D, eight-nodes, cohesive element (COH3D8) with an UMAT subroutine, which, while providing the constitutive relationship, could grant the possibility of damage initialization.

This approach has two main advantages. The first one is that the elements kinematics is managed by ABAQUS that, subsequently, calculates nodal displacements. The second, and most important one, is that damage variables used within the UMAT subroutine to define the cohesive constitutive behavior can be initialized according to the procedures that will be explained in the following sections.

Traction-separation ABAQUS cohesive elements are intended for bonded interfaces where the interface thickness can be considered small compare with laminae thickness. For this approach a linear relationship is established between traction vector $\tau$ and separation vector $\delta$ (also termed displacement jump), as follows:

$\boldsymbol{\tau}=\left\{\begin{array}{l}\tau_{n} \\ \tau_{s} \\ \tau_{t}\end{array}\right\}=\left[\begin{array}{lll}K_{n n} & K_{n s} & K_{n n} \\ K_{s n} & K_{s s} & K_{s t} \\ K_{t n} & K_{t s} & K_{t t}\end{array}\right]\left\{\begin{array}{c}\delta_{n} \\ \delta_{s} \\ \delta_{t}\end{array}\right\}$

where subscripts correspond to the three modes: opening $(\mathrm{n})$, shearing $(\mathrm{s})$ and tearing $(\mathrm{t})$ mode. The terms in matrix $\mathbf{K}$ must be intended as the equivalent stiffness coefficients of the cohesive element material, or, alternatively, $\mathbf{K}$ terms may be interpreted as element penalty stiffnesses for the three opening modes. In this meaning is typical to consider the modes as non-interactive and thus set to zero the off-diagonal terms.

Given the small thickness of the inter-laminar layer between plies, stratified composites fit well in the tractionseparation constitutive behavior, consequently we decided to develop a UMAT subroutine implementing the simplest possible traction-separation constitutive model that is the bi-linear one ${ }^{1}$.

The bi-linear law is one of the most used because it is based on few parameters that can be easily defined and identified. The relationships for the UMAT subroutine that allow mixed-mode response of the cohesive elements are based on the assumptions presented in [11, $12]$.

1 The damage initialization method developed in the paper can be extended to any constitutive cohesive law by modifying the UMAT appropriately. 
The key features of the cohesive constitutive model are briefly presented in this section, further details can be found in [12]. As proposed in [12], equal elastic constants may be assumed for the three opening modes so that the elastic part of the traction separation law becomes:

$$
\tau=K \delta
$$

this choice is justified by the following reasoning. Initial stiffness coefficients are must be high - so that the elastic properties of the laminate are unaffected - consequently, if $K$ identifies the highest of them the model is not very sensitive to the differences between $K$ and the real stiffness coefficients. In mixed mode conditions the traction-separation law is more conveniently expressed in terms of stress norm $\tau$ :

$\tau=\sqrt{\tau_{n}^{2}+\tau_{s}^{2}+\tau_{t}^{2}}$

and displacement jump norm $\lambda$ (alternatively termed equivalent displacement jump):

$\lambda=\sqrt{\left\langle\delta_{n}\right\rangle^{2}+\delta_{\text {shear }}^{2}} \quad \delta_{\text {shear }}=\sqrt{\delta_{s}^{2}+\delta_{t}^{2}}$

In the definition of $\lambda$ MacAuley brackets ${ }^{2}$ are used to neglect negative values of $\delta_{n}$ since contact between delaminated surfaces prevents inter-penetration. The mode-mixity is defined in terms of displacement jump components through parameter $\beta$ :

$\beta=\frac{\delta_{\text {shear }}}{\delta_{n}+\delta_{\text {shear }}}$

The bilinear cohesive law is shown in Figure 1, for a fixed mixed-mode ratio. The initial elastic response (curve AB) is defined by the penalty stiffness parameter. As traction reaches the interfacial strength of the material damage initiates (Point B). The penalty stiffness and the interfacial strength define the displacement jump at the onset of damage $\left(\lambda_{0}\right)$. If the displacement jump is increased beyond $\lambda_{0}$ the cohesive traction linearly decreases to zero (softening curve BD) and the initial stiffness is progressively decreased by the damage evolution law. When the displacement jump reaches a prescribed maximum value (point D), interface failure occurs and the interface load-bearing capacity vanishes. The area under the traction-separation curve represents the work needed to create a new delamination surface, that is the fracture toughness of the material at the considered mode-mixity. If at a given displacement jump (point $\mathrm{C}$ ) the load is reversed the constitutive behavior is linear (curve CA) with a stiffness that is decreased

\footnotetext{
2 Macauley brackets $\langle\cdot\rangle$ are defined so that: $\langle x\rangle=$ $(x+|x|) / 2$
}

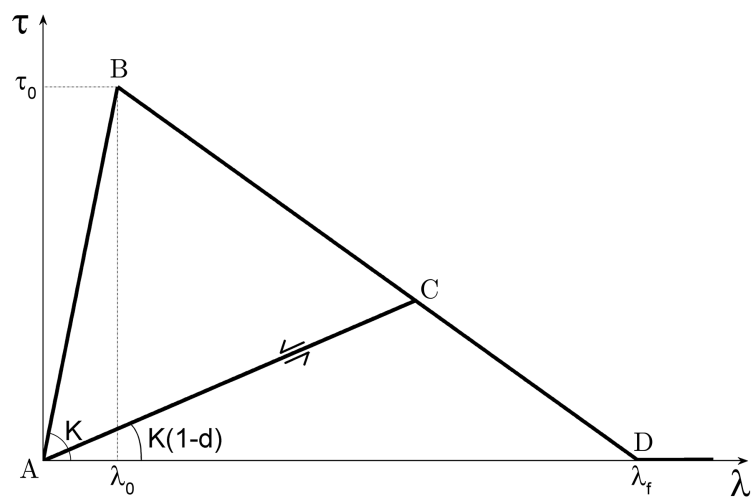

Fig. 1 Cohesive bi-linear constitutive law example at a given mode-mix.

according to the damage reached at point $\mathrm{C}$.

Damage onset is predicted by means of a quadratic criterion, with the further assumption of equal shearing and tearing strengths (i.e. $\tau_{s}^{0}=\tau_{t}^{0}=\tau_{\text {shear }}^{0}$ ):

$\left(\frac{\left\langle\tau_{n}\right\rangle}{\tau_{n}^{0}}\right)^{2}+\left(\frac{\tau_{\text {shear }}}{\tau_{\text {shear }}^{0}}\right)^{2}=1$

from which the onset displacement jump for any mode$\operatorname{mix}\left(\lambda_{0}\right)$ can be derived in terms of the onset displacement jumps of pure modes $\left(\lambda_{n}^{0}\right.$ and $\left.\lambda_{\text {shear }}^{0}\right)$. For values of $\lambda$ greater than $\lambda_{0}$ a scalar damage variable $d$ can be defined to model the softening part of the tractionseparation law. Variable $d$ represents the effects of the interlaminar damage mechanisms which progressively reduce the interface stiffness after onset conditions are reached. When damage is active the constitutive relationship becomes:

$\boldsymbol{\tau}=K(1-d)\left\{\begin{array}{l}\delta_{n} \\ \delta_{s} \\ \delta_{t}\end{array}\right\}-d\left\{\begin{array}{c}\left\langle-\delta_{n}\right\rangle \\ 0 \\ 0\end{array}\right\}$

The second term on the right hand side of equation (7) prevents damage, and the degradation of stiffness, when sublaminates are in contact (i.e. for negative values of the normal displacement jump).

Since damage is an evolutionary irreversible process, damage rate must be non-negative. In cohesive elements damage remains constant (i.e. damage rate is zero) when displacement jumps diminish and tractions decrease (unloading). As a consequence, for generic load histories a correct evaluation of damage onset and evolution requires the definition of internal variables an algorithms that distinguish loading and un-loading phases and describe damage evolution in rate form $[11,12]$.

The loading/un-loading status is captured by the time derivative of the equivalent displacement jump $\dot{\lambda}$, while internal variable $r(t)$ is defined to store the damage 
threshold. By definition, $r=\lambda_{0}$ for an undamaged condition.

By using $r$ we may say that damage does not change as long as $\lambda(t) \leq r(t)$ or equivalently [11,12] as long as the following holds true:

$f(\lambda(t), r(t)):=g(\lambda(t))-g(r(t)) \leq 0$

with $g(\alpha)$ a monotonic function of its argument that ranges between zero, when the argument takes the value $\lambda_{0}$, and one. For a generic load history with loading and un-loading phases, functions $g$ and $f$ define the evolution of damage through damage rate equations (see $[11$, 12] for details):

$\dot{d}=\dot{\mu} \frac{\partial g(\lambda)}{\partial \lambda} \quad \dot{\mu}=\dot{r}$

and the Kuhn-Tucker relations:

$\dot{\mu} \geq 0 ; \quad f(\lambda, r) \leq 0 ; \quad \dot{\mu} f(\lambda, r)=0$

with $\mu$ an internal variable of the model.

Equations 9 and 10 can be integrated to obtain that: damage threshold at current time $t$ equals either $\lambda_{0}$ or the maximum value reached by $\lambda$ at any time $s \leq t$, whichever is the greatest, while damage is given by the value function $g$ takes for the current value of damage threshold, i. e. $d(t)=g(r(t))$.

In order to have a traction-separation law that, for a given mixed-mode ratio $(\beta=\bar{\beta})$, has a linear softening, the damage evolution law can be defined as:

$g(r)=\frac{\lambda_{f}(\bar{\beta})\left[r-\lambda_{0}(\bar{\beta})\right]}{r\left[\lambda_{f}(\bar{\beta})-\lambda_{0}(\bar{\beta})\right]}$

Thus, the damage evolution law is identified through onset displacement jump $\lambda_{0}$ and failure displacement jump $\lambda_{f}$ : for $r \geq \lambda_{f}$ damage reaches one and the stiffness of the cohesive layer vanishes ${ }^{3}$.

Interlaminar failure, at any mode-mix, is assumed to occur when the energy release rate $G$ reaches a critical value $G^{c}$. For pure modes the critical energy release rate coincides with modal fracture toughness: $G_{n}^{c}$ for the opening, $G_{s}^{c}$ for the shearing and $G_{t}^{c}$ for the tearing mode. Under mixed-mode loading the critical energy release rate is given as a function of pure modes fracture toughness and of the energy release rate components. Among the number of different expression that

\footnotetext{
3 In order to avoid numerical problems during implicit simulations that do not allow the removal of completely damaged elements, a very small stiffness is retained. Dedicated checks of the results can easily confirm that such residual stiffness does not affect the results of the analyses.
}

are available, the one proposed by Benzeggagh and Kenane $(\mathrm{BK})$ in $[26]$ will be used in this work under the assumption that $G_{t}^{c}=G_{s}^{c}$ :

$G_{e q}^{c}(B)=G_{n}^{c}+\left(G_{s}^{c}-G_{n}^{c}\right)(B)^{\eta}$

where mode-mixity is identified through parameter $B$, that is defined as:

$B=\frac{G_{\text {shear }}}{G}$

with $G_{\text {shear }}=G_{s}+G_{t}$ and $G=G_{n}+G_{\text {shear }}$. The values of toughness $G_{n}^{c}$ and $G_{s}^{c}$ and of mixed-mode interaction parameter $\eta$ can be evaluated by means os standard tests such as Double Cantilever Beam (DCB) [27], End-Notched Flexure (ENF) and Mixed Mode Bending (MMB) [28].

The value of failure displacement jump $\lambda_{f}$ is finally computed observing that, for a given mixed-mode ratio, the area under the traction-separation curve represents the interlaminar critical fracture energy, thus:

$\lambda_{f}(B)=\frac{2 G_{e q}^{c}(B)}{K \lambda_{0}}$

Since $B$ is defined as a ratio of energy release rates for the current displacement jump, a relation can be established [12] between $B$ and $\beta$ which reads:

$B=\frac{\beta^{2}}{1-2 \beta+2 \beta^{2}}$

The bilinear constitutive law proposed may become thermodynamically inconsistent, for a generic, arbitrary, set of model parameters, when mode-mix changes during a simulation [15]. Following [15], such possibility is removed if a relation is enforced between inter-laminar strengths and energy release rates of pure modes:

$\tau_{n}^{0}=\tau_{\text {shear }}^{0} \sqrt{\frac{G_{n}^{c}}{G_{s}^{c}}}$

Although this choice might seem limiting, we believe the model is still generally applicable especially for brittle materials, like composites, for which the inter-laminar damage process is essentially dominated by fracture energy release rates. In this cases, the inter-laminar strengths can be artificially lowered ([13] and references therein) in order to expand the process zone and, consequently, increase the size of the elements required to capture the stress field in the area ahead of the delamination front $[13,19]$.

Moreover, for laminated composites the inter-laminar normal strength is difficult to measure, thus it may be conveniently estimated from its shear counterpart by means of equation (17). 
As far as model tuning is concerned, special care must also be devoted to the selection of the penalty stiffness since it may have a negative impact on simulation performance. Penalty stiffness choice requires a trade-off between the necessity to have a stiff connection between adjoining elements, so that the initial elastic properties of the laminate are unaffected, and the opportunity to minimize numerical troubles, with the associated increases in the computational time. Indeed, high values of penalty stiffness combined with the discontinuity of the tangent stiffness matrix at damage initiation can cause spurious traction oscillations [25].

Tangent stiffness matrix discontinuities may also cause convergence problems with implicit integration schemes, especially when used in combination with algorithms that control time increments automatically [18]. A mitigation of such problems is offered by numerical viscous regularization that may applied to the damage evolution. It basically consists in replacing damage variable $d$ in equation (7) with its regularized counterpart given by:

$\dot{d}_{v}=\frac{1}{\eta}\left(d-d_{v}\right)$

with $d$ calculated according to equations (8-11) and $\eta$ indicating the artificial viscosity of the model. Naturally, values of $\eta$ must be selected small enough so that they generate negligible effects in the results of the simulations.

\section{The multi-step approach and its implementation}

The proposed approach is based on the ABAQUS subroutine SDVINI, which can be invoked by an UMAT subroutine, at runtime, to define initial values of internal variables (STATEV). Figure 2 shows a work-flow of an ABAQUS multi-step implicit analysis that uses of SDVINI and UMAT subroutines.

Step 0 is a default step of any analysis (single- or multistep). ABAQUS uses Step 0 to perform internal actions needed to start the user-defined steps that follow. In particular, the SDVINI subroutine can be used to define the initial values of any of the STATEVs (i.e. damage variables), which are then passed to the UMAT subroutine. The SDVINI subroutine is invoked once for each integration point of each element characterized by an user-defined material. The initialization via SDVINI can exploit either the coordinates of the integration point or the internal element number. Once the initial state is defined, the analysis starts and the evolution of the initial state is controlled by the UMAT subroutine, which, in each iteration, receives from ABAQUS the strain components, computes the new values of the damage variables, updates the element stiffness matrix and returns to ABAQUS the stress components and the jacobian matrix. The following subsections describe the three proposed approaches to damage initialization via SDVINI.

\subsection{Geometrical initialization}

In this approach two distinct modes can be used to obtain an analytically defined domain where a damage variable is initialized to a prescribed value. In the simplest approach the domain is identified through geometrical coordinates within analytically defined boundaries; this requires the FE model be referred to a convenient coordinate system. A more efficient and flexible method to obtain analogous results consists in creating, within ABAQUS graphic user interface (CAE), a set that includes all the elements in the domain to be initialized. Elements in such set share the same usermaterial and are initialized by the SDVINI. The drawback is that the UMAT is more complex since one must define multiple internal cases to cope with user-materials in different states (pristine or damaged). The main advantage is that CAE can be advantageously used to define domains with more general shapes.

\subsection{NDI-like initialization}

In principle, the method which uses internal sets can initialize delaminations with arbitrary shapes reconstructed from Ultrasonic C-scanning (CSCAN) images but this can be extremely user-intensive and time consuming. A smarter approach, based on automatized image analysis, has been developed and it consists in the following key steps:

- the CSCAN images are acquired and transformed in binary black and white images to identify the delaminated areas;

- external dedicated scripts are used to gather the value of each pixel ( 0 for delaminated areas and 1 otherwise) and their normalized coordinates (one matrix for each inter-laminar layer);

- SDVINI reads the damage matrices and converts normalized coordinates into real ones to initalize damage variables in the UMAT subroutine.

This initialization technique needs a pre-process step to be performed: the delaminated shapes are positioned within a reference frame of known dimensions (width and height) and distinct raster files are generated for 


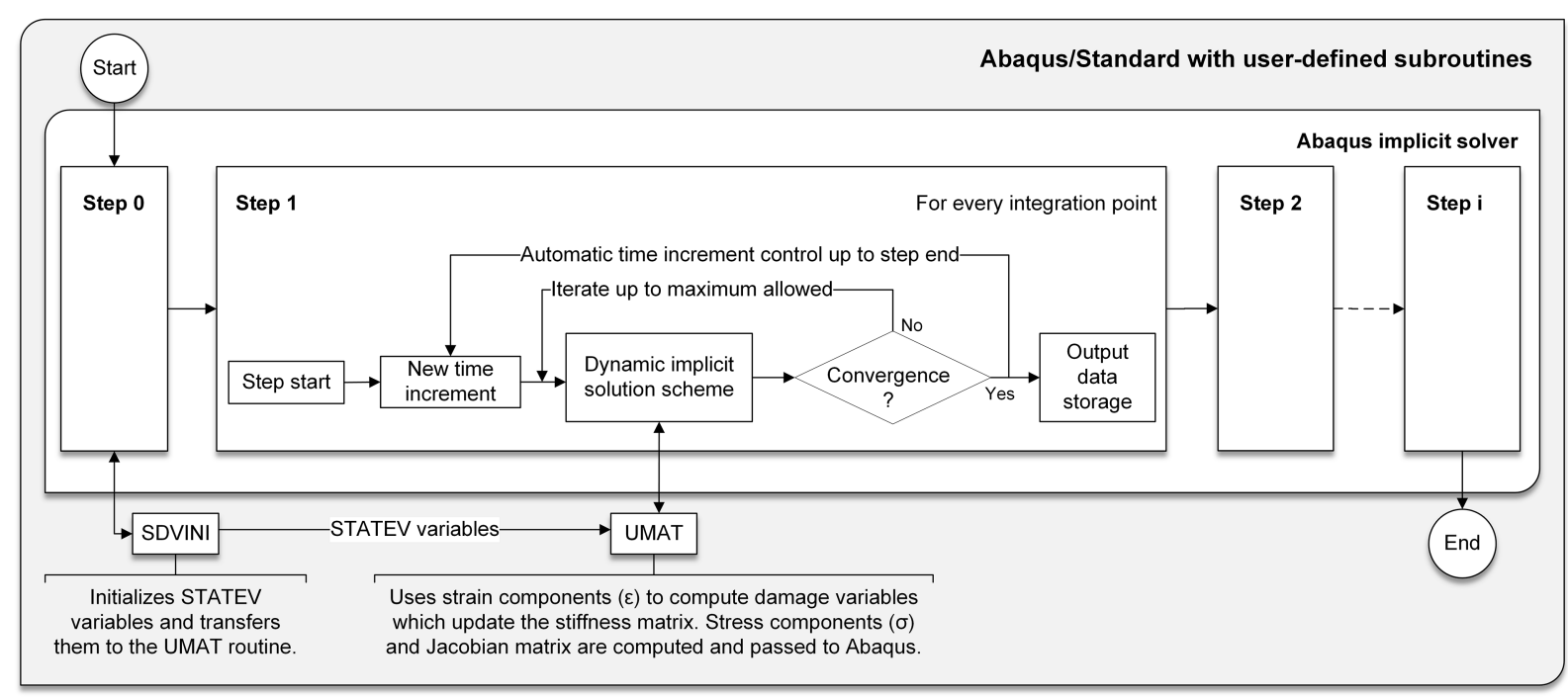

Fig. 2 Schematic flowchart of a multi-step analysis in which SDVINI and UMAT subroutines are used.

each interface. In this step, the image resolution is chosen in order to reproduce well the delamination fronts. It is worth highlighting that even if high resolution image are generated, the actual resolution of the initialized delamination fronts is ruled by the FE discretization. For each image, the delaminated and the undamaged areas are filled with uniform colors, black and white respectively. The latter operation is carried out to extract and store into matrices (one per interface) the normalized coordinates of the pixel vertices that are within the delaminated areas. Normalized coordinates are computed by dividing each component ( $\mathrm{x}$ and $\mathrm{y}$ ) for the relevant reference length (width and height of the reference frame). Eventually, the matrices are saved into a ASCII file, which is read by the SDVINI subroutine.

\subsection{Simulation-based initialization}

This approach has been developed in order to recreate a complex damage scenario, within a large aerospace structure. Two distinct FE simulations are performed: in the first one interlaminar damage is produced in a FE model of a representative structure, then damage is injected into a pristine model of the same structure. This application uses identical FE models for the analyses, at least locally, even though this limitation can be removed in future developments. The transfer of damage information between the two analyses is controlled by two Python scripts, described as follows:

- a first script is used to access to the output database file (.odb), produced by the first analysis, which stores the STATEV damage variables, internal el- ement IDs ${ }^{4}$ and integration point coordinates. The script save the relevant data into external ASCII files for future use;

- the second script reads damage information from external ASCII files and organizes them according to element IDs or integration point coordinates. The reordering of the damage information is carried out considering specific details ${ }^{5}$ of the second FE analysis. The sorted data are vital to efficiently perform the initialization of the second analysis especially for large models;

- finally, SDVINI reads the sorted data and initializes the requested damage variables in the second analysis.

\section{Results and discussion}

In this section the results relevant to the following testcases are presented:

1. a simple geometrical initialization of a circular artificial damage to simulate delamination-buckling under compression;

2. a NDI-like initialization test-case in which multiple measured delaminations are injected into a composite plate subjected to axial compression (in CAI-test like set-up);

3. a simulation-based initialization test-case where delaminations are produced in a stiffened panel via

4 ABAQUS CAE assigns unique element IDs within a part instance, only at runtime unique element IDs are assigned within the whole model.

5 In the current version the two models need to be identical in the areas interested by damage but can differs in terms of active part instances and boundary conditions. 
static indentation and then injected in a compressionbuckling analysis that causes delamination growth.

\subsection{Geometrical initialization test-case}

The developed inter-laminar constitutive behavior, implemented in a dedicated UMAT subroutine, has been assessed by comparison with the results of a Compression After Impact (CAI) test of a carbon/epoxy specimen with a circular defect manifactured with a teflon insert [21]. The specimen has a $\left[(-45 /+45 / 90 / 0)_{2} /-\right.$ $60 /+60 /-15 /+15]_{s}$ lamination with the teflon insert between the $5^{\text {th }}$ and $6^{\text {th }}$ ply $(-45 /+45$ interface). This specific test-case has been chosen due to the following factors: a relatively simple defect within the laminate, i.e. circular delamination, and a good set of experimental data in terms of force applied, deformation and propagation of delamination. Moreover the numerical results are also compared with the ones reported in [18], for the same test-case, where the Virtual Crack Closure Technique (VCCT) is used. Figure 3 shows the boundary conditions enforced in the Finite Element model together with an example of how the plate has been modeled: two sub-laminates with a mesh size of $0.5 \mathrm{~mm}$, modeled with three continuum shell elements $(S C 8 R)$ for the thicker sub-laminate and one element for the thinner one, and layer of cohesive elements (COH3D8 elements) with a thickness of $0.01 \mathrm{~mm}$ to simulate the propagation of the pre-existing delamination under compressive load. The numerical values used to characterize the cohesive behavior are: $t_{n}^{0}=30$ $\mathrm{MPa}, t_{s}^{0}=t_{t}^{0}=70 \mathrm{MPa}, K=150000 \mathrm{MPa} / \mathrm{mm}$. Three $\mathrm{FE}$ models with the same rectangular FE discretization of the sub-laminates but different cohesive mesh layers, Figure 4, have been created:

- the first cohesive layer (Rad-H), Figure 4 - left, has a radial mesh with no cohesive elements within the area of the defect in order to reproduce the presence of the initial defect (contact interactions between the facing sub-laminates enforced);

- the second cohesive layer (Rad-I), Figure 4 - center, has a radial mesh similar to the first cohesive layer but the defect is reproduced via geometric initialization (no need for both contact interactions and elements deletion within the defect area);

- the third cohesive layer (Rect-I), Figure 4 - right, shares the same mesh of the adjacents sub-laminates and the circular defect is obtained via initialization.

Due to the diversity of the FE discretization between the cohesive layer and the sub-laminates, a TIE constraint is enforced for the models Rad-H and Rad-I while the model Rect-I is created by merging the superposed nodes. For each model, a maximum radius, equal to $52.0 \mathrm{~mm}$ in which damage can propagate has been set (annulus around the initial defect in Figure 4). This value has been estimated considering the maximum delamination length, measured via X-rays, reported in [21]. Dynamic implicit simulations of the CAI tests have been performed in ABAQUS with an imposed compressive displacement of $1.0 \mathrm{~mm}$ in $60 \mathrm{~s}$. In each FE model a geometrical imperfection with a maximum out-of-plane displacement of the thin sub-laminate equal to $0.1 \mathrm{~mm}$ (as suggested in [21]) is introduced. The shape of the geometrical imperfection is extracted by dedicated linear buckle simulations for each model. The FE models are provided with axial connectors in specific locations which have been used to reproduce the strain gauges readings. Axial connectors provide an output used to promptly compute strain along a specific direction (the connector direction). The axial strains are, thus, evaluated in a position (far field strain in [21]), along the center line of each model, where the influence of both the initial delamination and the fixture is negligible. Figure 5 shows the compressive load vs. the axial strain and the delamination length vs. the compressive load of both the experimental test and the simulations. The delamination length for increasing loads is measured along the major axis of the growing delamination, highligthed in Figure 5(right). The delamination propagates along an inclined direction (the same angle for all the FE models) due to the different orientation of the adjacent plies $(-45 /+45)$ and it is qualitatively similar to the experiments (no quantitative data are reported in [21]). The models with the radial mesh ( $R a d-H$ and $R a d-I)$ match the experimental force vs. axial strain, Figure 5 (left), up to approximately $62 \mathrm{kN}$ while the model with the rectangular mesh (Rect- $I$ ) continues with a good approximation up to almost $70 \mathrm{kN}$. The three numerical curves are able to capture the experimental data better than the results obtained with the VCCT analysis.

The differences between the results can be explained looking at the delamination length vs. applied load comparison reported in Figure 5 (left). Models $R a d-H$ and Rad- $I$ show a delamination growth quite close to the experimental data for loads lower than $60 \mathrm{kN}$ while model Rect-I shows a lower delamination grow-rate and a better match of the experimental data up to almost $70 \mathrm{kN}$. Each analysis is stopped when the front of the delamination reaches the limit for delamination propagation showed in Figure 4.

The results confirm that the geometrical initialization procedure is capable to correctly initialize inter-laminar damage by exploiting the spatial coordinates of inte- 

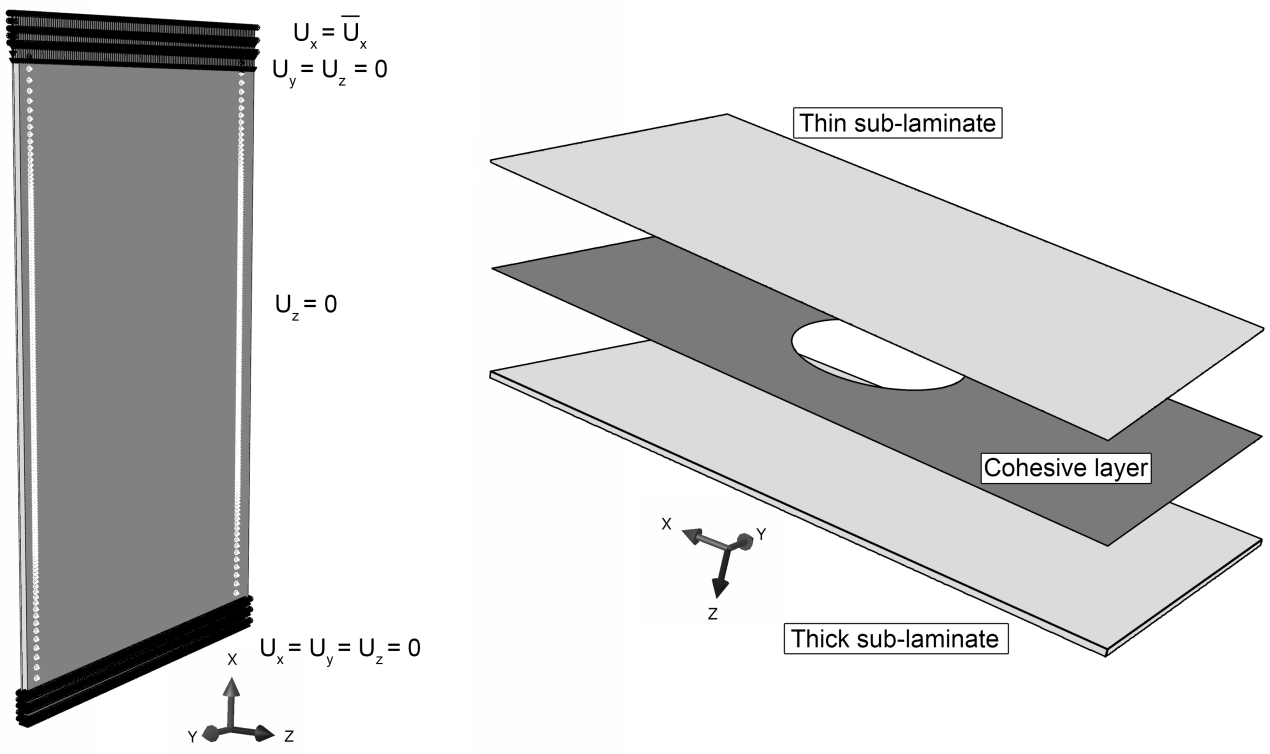

Fig. 3 Geometrical initialization test-case boundary conditions (left) and exploded view of the sub-laminates and of the cohesive layer (right).
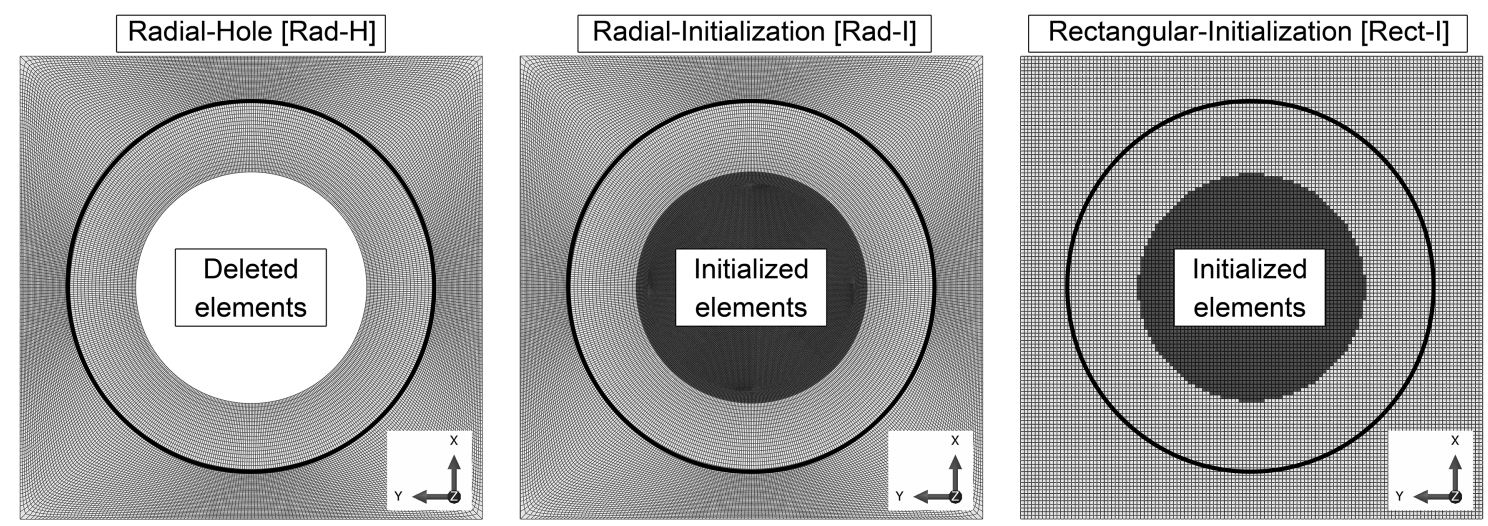

Limit for delamination propagation

Fig. 4 FE models of the cohesive layer for the delamination buckling analyses.

gration points. A first verification of the proposed initialization procedure stems from the consideration that equal results are obtained for the models with the same radial mesh ( $R a d-H$ and $R a d-I$ ). In this case the initialized model overcomes the necessity to delete elements within the defect area and to enforce contact interactions between the facing sublaminates. The results also highlight how the proposed initialization can foster a time-saving modeling procedure since it can be easily applied to non-tailored mesh (as the rectangular one) which does not require the cohesive layer be partitioned or elements be deleted. An accurate investigation of the effect of the different parameters that characterize the current damage model to predict the onset of delamination, its growth rate and the shape of the growing delamination is beyond the scopes of the current work and further studies are ongoing. In particular, it is worth highlighting the differences, in terms of delamination growth, that arise between the models with the cohesive radial mesh, tied to the adjacent sublaminates, and the model with the cohesive rectangular mesh, connected by merging coincident nodes, for loads above $60 \mathrm{kN}$.

\subsection{NDI-like initialization test-case}

The proposed initialization method has been applied in a Compression After Impact (CAI) simulation for which experimental data of positions, shapes of delaminated areas and values of residual compressive strength of car- 

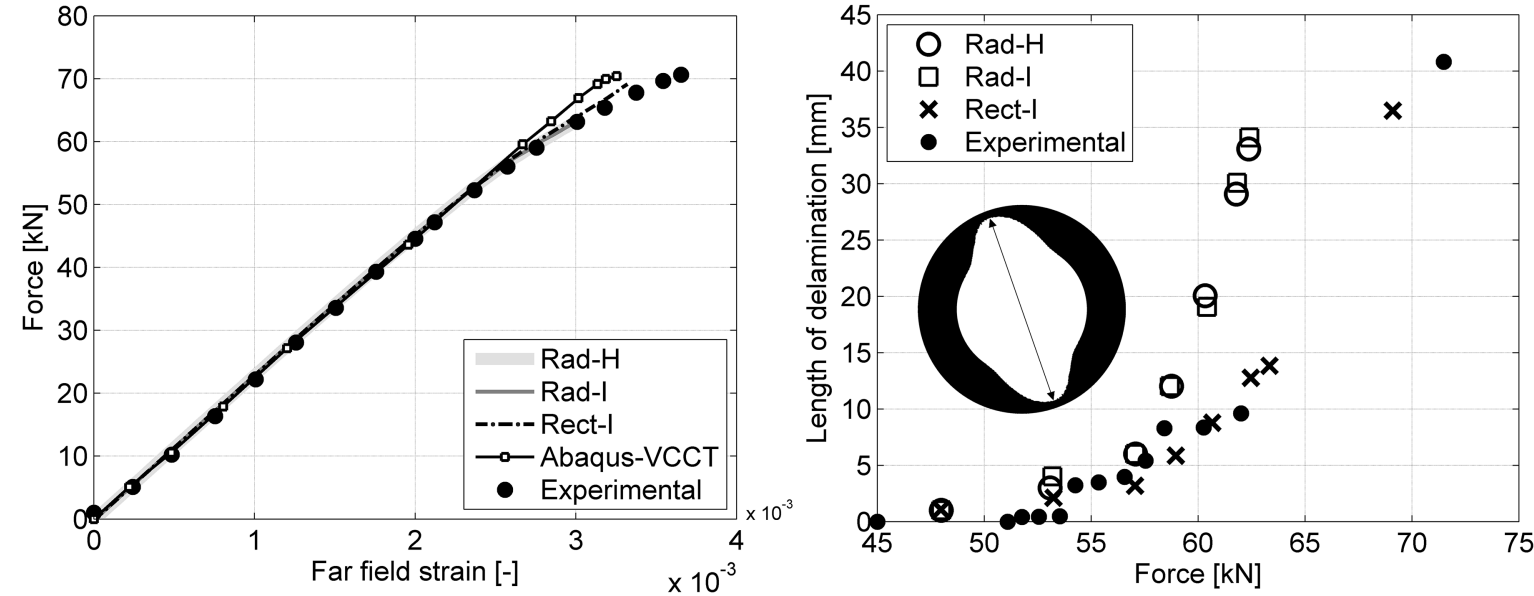

Fig. 5 Applied load vs. far field strain (left) and length of delamination vs. applied load (right).
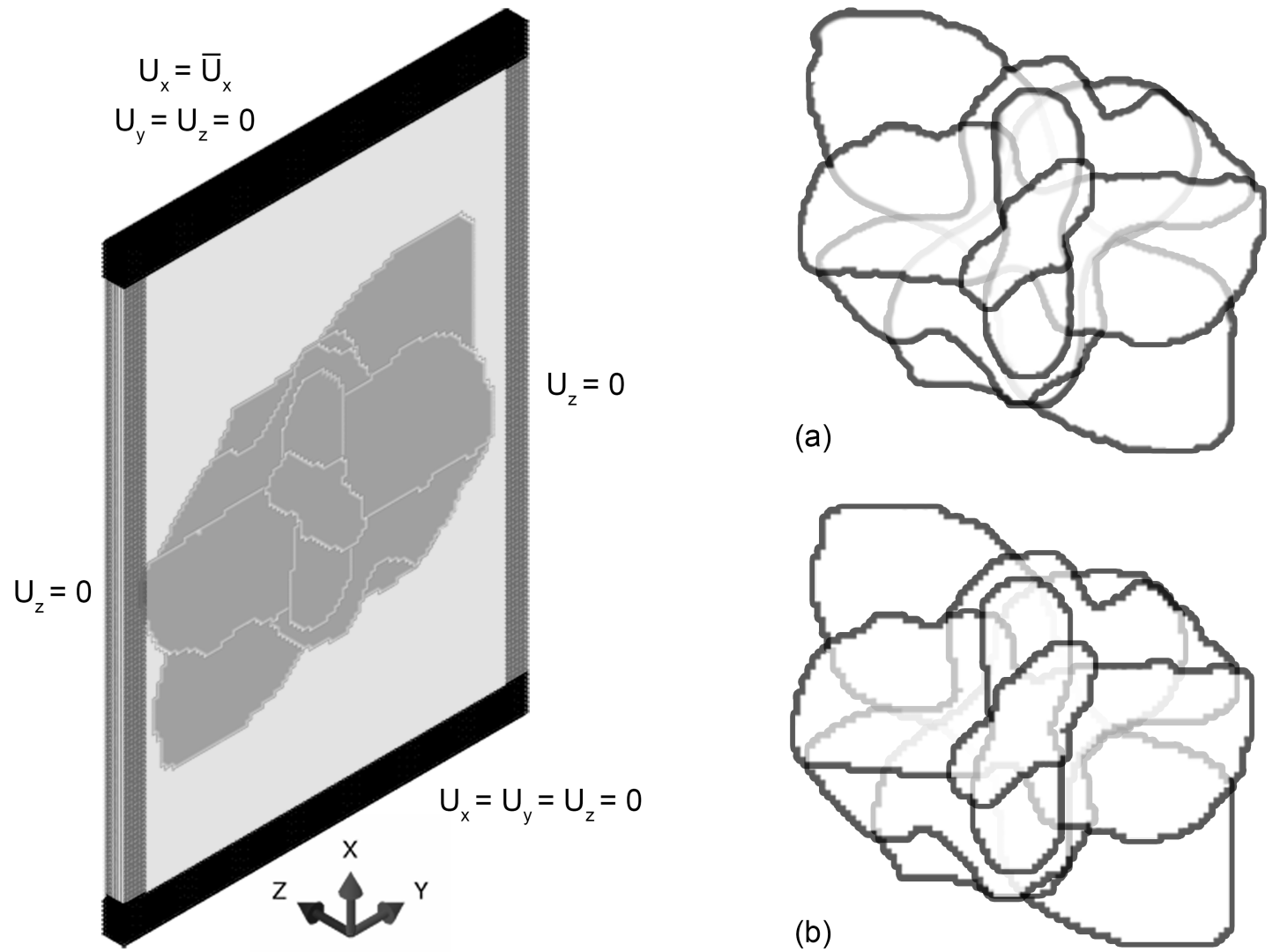

Fig. 6 Boundary conditions of the NDI-like initialization test-case (left) and delamination shapes (right): C-SCAN reconstructed shapes (a) and initialized ones (b). 


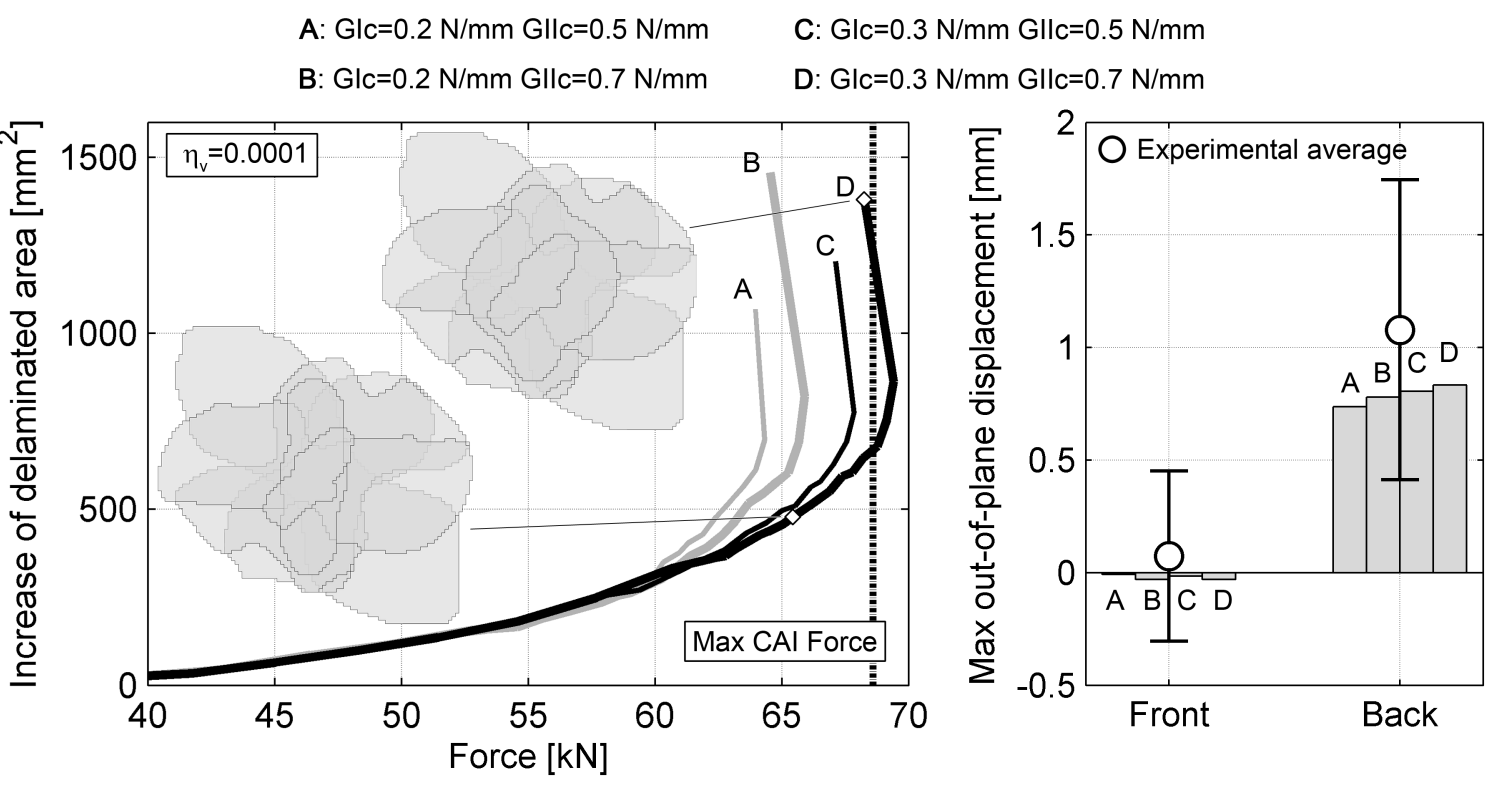

Fig. 7 Increase of delaminated area vs. compressive load (left) and maximum out-of-plane displacement (right).

bon/epoxy laminate are available in [22]. The specimen is a $150 \mathrm{~mm} \times 100 \mathrm{~mm}$ plate with a $\left[-45_{4} / 45_{4} / 0_{3} / 90\right]_{s}$ laminate and a total thickness of $4.53 \mathrm{~mm}$. The relevant elastic material properties can be found in [22]. The position and the shape of delaminations resulting from impact events (approximately $10 \mathrm{~J}$ ) are obtained through ultrasonic inspections which show that the delaminations occurred in correspondence of change in ply orientation, thus, six delaminated interfaces are visible. Dynamic implict analyses are performed to evaluate both the maximum CAI force and the maximum out-of-plane displacement for which experimental data are available in [22]. The load is introduced by imposing a compressive displacement of $2 \mathrm{~mm}$ in $60 \mathrm{~s}$. A sensitivity analysis on the Mode I and Mode II fracture toughness values used to characterize the constitutive behavior of cohesive elements is carried out since no data for the selected material were available. The Finite Element model is shown in Figure 6. The laminate has been reproduced by gathering together each group of plies that share the same orientation in continuum shell elements $(S C 8 R)$ spaced out by six inter-laminar layers of cohesive elements $(C O H 3 D 8)$. Each cohesive layer is modeled with a physical thickness of $0.01 \mathrm{~mm}$. The constitutive parameters used to characterize the bilinear constitutive law are: $t_{n}^{0}=15 \mathrm{MPa}, t_{s}^{0}=t_{t}^{0}=30$ $\mathrm{MPa}, K=150000 \mathrm{MPa} / \mathrm{mm}$. A small value (0.0001) of numerical viscosity is used to foster faster convergence. Figure 6 also shows the applied boundary conditions and the shapes of the delaminations captured from [22] (a) and the initialized ones (b), which present jagged edges due to the FE discretization. Since only projected delamination areas are available in [22], some a priori assumptions have been made for the not visible delamination fronts.

Eventually, since experimental CAI tests were performed on impacted specimens for which average maximum permanent indentation measurements were available $(0.29$ $\mathrm{mm}$ ), each CAI simulation has been carried out with the same initial imperfection.

Figure 7 shows the increase of the overall delaminated area (sum of the delaminated area of each cohesive layer) vs. the applied compressive load and the experimental maximum CAI force obtained in the experiments. The evolution of the initialized delamination scenario is the same for all the simulations (an example of delaminations for two different loads is shown in Figure 7 (left)). The propagation of delaminations in each simulation is stable up to the maximum force. Afterward instable propagation is exhibited by each model and the analyses are interrupted due to low time increments. A good agreement in terms of maximum force exist between the experiments and the simulations $C$ and $D$. The comparison of the out-of-plane displacement, Figure 7 (right), shows that the numerical results are within the error band of the experiments but slightly below their average values. The labels Back and Front are referred to the side of the specimen opposite to the impactor and the impactor side, respectively. The impact deflection direction is considered as positive displacement.

The proposed initialization procedure has proven to be able to initalize multiple delaminations on the basis of 


\begin{tabular}{ccc}
\hline \multicolumn{4}{c}{ Elastic constants } \\
\hline$E_{11}$ & 112.7 & {$[\mathrm{GPa}]$} \\
$E_{22}=E_{33}$ & 10.35 & {$[\mathrm{GPa}]$} \\
$G_{12}=G_{13}$ & 3.50 & {$[\mathrm{GPa}]$} \\
$G_{23}$ & 3.64 & {$[\mathrm{GPa}]$} \\
$\nu_{12}=\nu_{13}$ & 0.32 & {$[-]$} \\
$\nu_{23}$ & 0.42 & {$[-]$} \\
\hline
\end{tabular}

Table 1 Lamina properties of the simulation-based test-case.

ultrasonic inspection data, allowing numerical residual strength analyses to be performed in CAI simulations.

\subsection{Simulation-based initialization test-case}

The simulation-based initialization procedure is assessed by means of a simulation on a carbon/epoxy stiffened panel. The panel is initially damaged (delaminations) through a steel hemispherical indenter and then axially loaded in compression to induce delamination growth. The panel is $150 \times 250 \mathrm{~mm}$ with a skin of $1 \mathrm{~mm}$ thick and two stringers with a reversed T-shape section with a flange thickness of $3 \mathrm{~mm}$.

The skin is a $[+45 /-45 / 0 / 90]_{s}$ laminate modeled with two continuum shell (SC8R) elements along the thickness. The relevant elastic material properties used to characterize the laminates are reported in Table 1. The stringers have a quasi-isotropic lamination and a substantial thickness in order to force a skin buckling behavior so that the propagation of delaminations during the compression step could be induced. The steel hemispherical indenter has a radius of $6.35 \mathrm{~mm}$ and is pressed against the skin with a displacement of $0.8 \mathrm{~mm}$ in $60 \mathrm{~s}$. An area in correspondence of the indentation site of the stiffened FE panel model is partitioned in order to introduce a ply-by-ply model of the skin where interposed cohesive layers (COH3D8 elements) can simulate delaminations within the skin laminate. An additional cohesive layer simulates the skin-stringer interface. For this reason also the stringer is partitioned and the created partition is meshed with the same mesh size of the adjacent cohesive layer. The partitioned portion of the model is tied to the surrounding model which has a coarser mesh. Details of the FE model of the indentation site are presented in Figure 8 (left). In order to better highlight the modeling details of this region a mixed wireframe-full mesh visualization has been devised. The resulting FE model contains in approximately 150000 elements.

The bilinear cohesive constitutive law is characterized by $t_{n}^{0}=15 \mathrm{MPa}, t_{s}^{0}=t_{t}^{0}=30 \mathrm{MPa}, K=150000$
$\mathrm{MPa} / \mathrm{mm}$. The inter-laminar fracture properties (Mode I and Mode II) were $G_{I c}=0.2 \mathrm{~J} / \mathrm{m}^{2}, G_{I I c}=G_{I I I c}=$ $0.7 \mathrm{~J} / \mathrm{m}^{2}$.

Dynamic implicit analyses are performed on a 12 cores workstation with two Intel Xeon E5645 2.40GHz processors and 32 GB ram. Two analyses are then performed: the first is a three-steps sequential analysis in which the indentation step is followed by a step to damp residual vibrations and the final compression one (CAI), the second uses the damage obtained at the end of the indentation step of the sequential analysis to initialize damage and to perform a stand-alone analysis of the panel compression. Figure 8 shows a scheme that summarizes the key points of both the sequential analysis (top in Figure 8) and the initialized one (bottom in Figure 8). The scheme points out each step of the two simulations, and highlights the damage data (delaminations), that are extracted from the indentation step (step 1 of the sequential analysis) and transferred to the one to be initialized. These data are internal state variables computed within the UMAT subroutine in each increment, for each element, at each integration point. It is important to highlight that, as long as the local model (the one with the interlaminar layers) does not change, the initialization procedure can initialize damage even if the global model, that surrounds the local one, is changed.

Figure 9 shows the boundary conditions enforced to the model. The displacement imposed to the indenter in the indentation step ( $B C$ type $B$ in Figure 9 ) and to one side of the stiffened panel in the compression step ( $B C$ type $C$ in Figure 9 ) are introduced via reference points connected through multi-point constraints to the corresponding model parts.

The two simulations are then compared in terms of inplane areas and shapes of the delaminations at the end of the compression step together with the out-of-plane displacement in order to show that a identical buckling patterns are followed by the two models. Figure 10 shows the delaminated shapes at the end of the indentation step and at the end of the compression one of the sequential analysis. Most of the propagation of the delaminations induced during the indentation step takes place in the interface between skin and stringer due to the stiff support of the thick stringer compared to the thin skin.

The reproducibility of the results obtained with the sequential analysis my the initialized one is moreover assessed by comparing the out-of-plane displacement $(U 3)$ for a similar applied compressive load in Figure 11 and by observing the similar growth, during the compression step, of the in-plane delaminated area of both the sequential and initialized simulation in Figure 12 


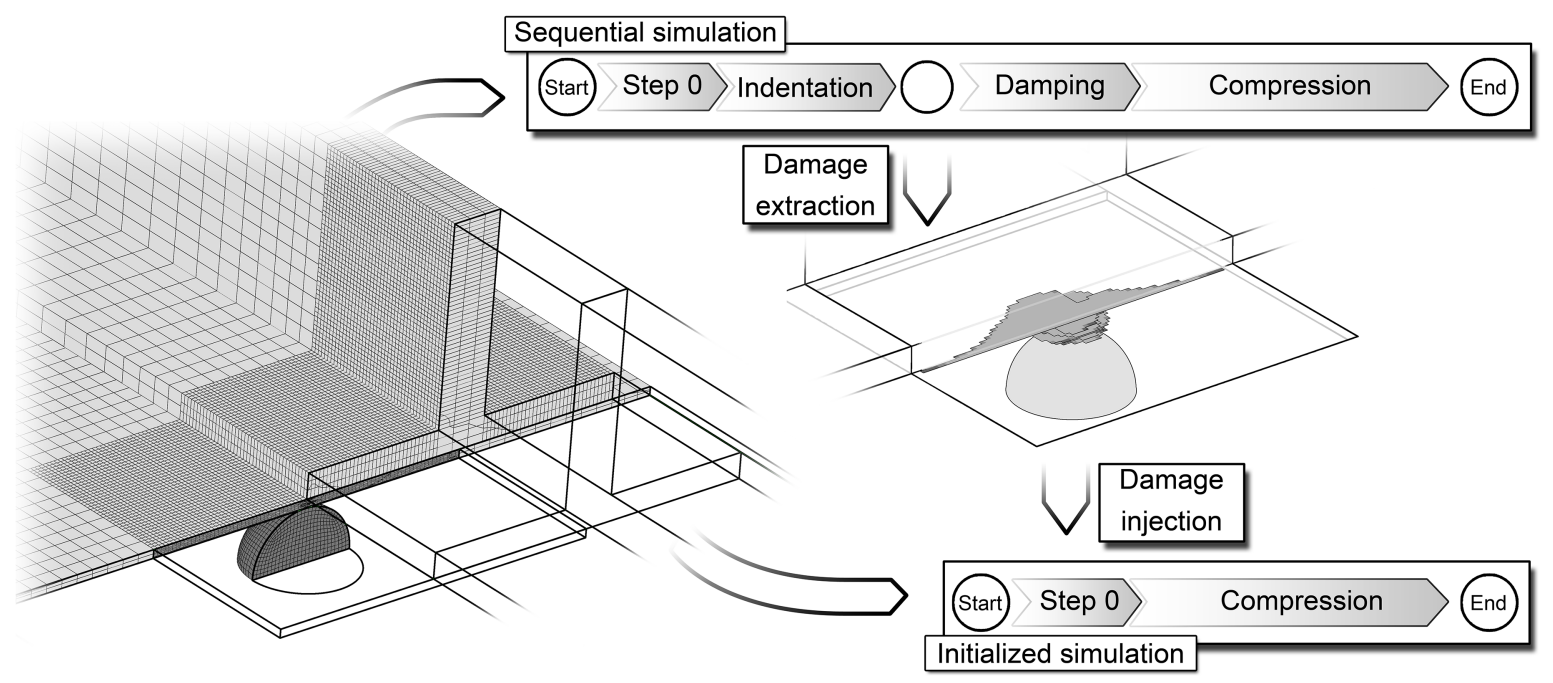

Fig. 8 Indentation site mesh detail and overview of the steps of the sequential analysis and of the initialized one.

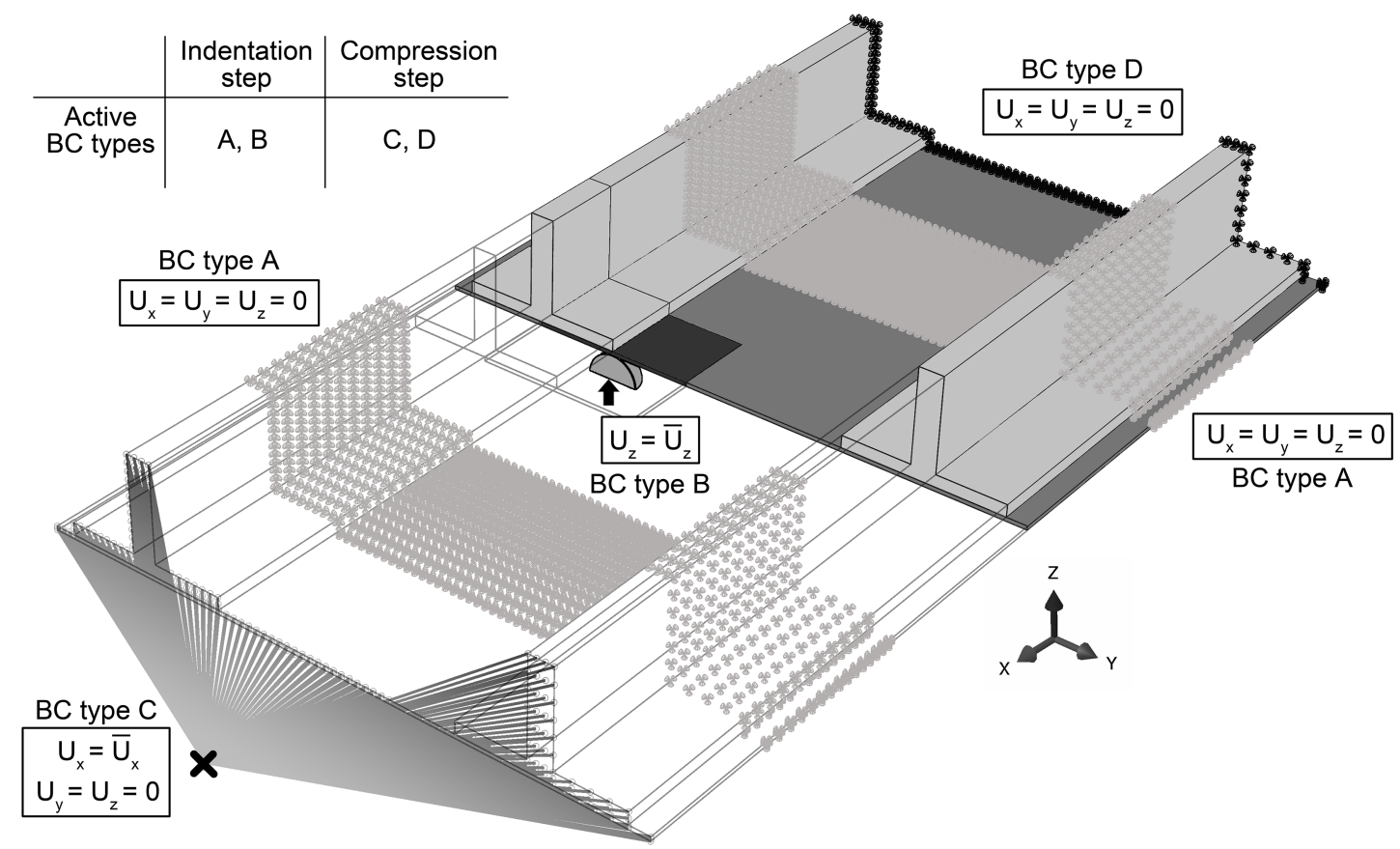

Fig. 9 Summary of all the enforced boundary conditions (a different set of conditions is used for each step).

(right). Figure 12 (right) has been created computing the in-plane delaminated area for similar values of compressive loads. Since the two simulations have distinct increment sequences, outputs are available for slightly different compressive load. Nonetheless, the delamination growth is identical and the final values are the same for the two simulations. Both the analyses are stopped when any delamination reaches the boundary of the local model. Eventually, Figure 12 (left) also shows the delaminated shape at the end of the compression step of the initialized analysis. The comparison between this shape and the one obtained for approximately the same compressive load in the sequential analysis, Figure 10 (right), reveals that also the propagation of the delamination is perfectly reproduced.

The obtained results show that the simulation-based initialization procedure is able to correctly initialize inter-laminar damage, extracted from a previous anal- 

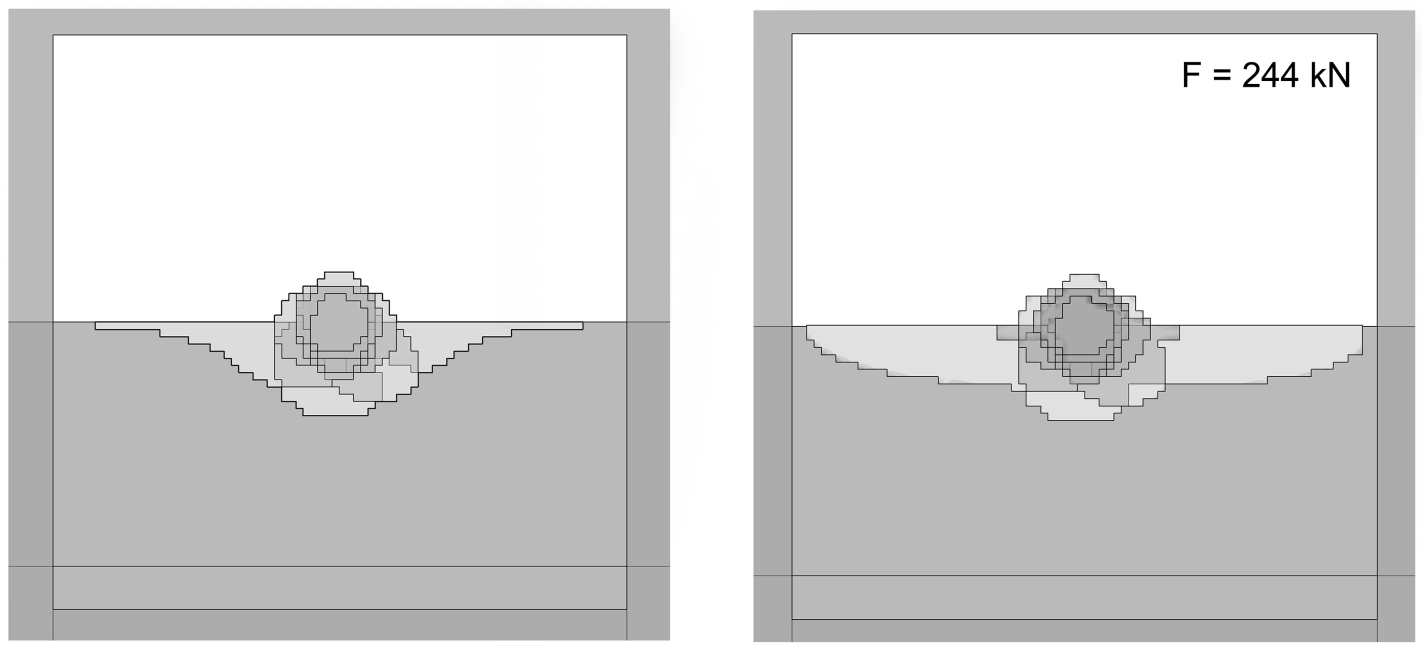

Fig. 10 Comparison of the delaminations pattern of the sequential analysis at the beginning (left) and at the end (right) of the compression step.

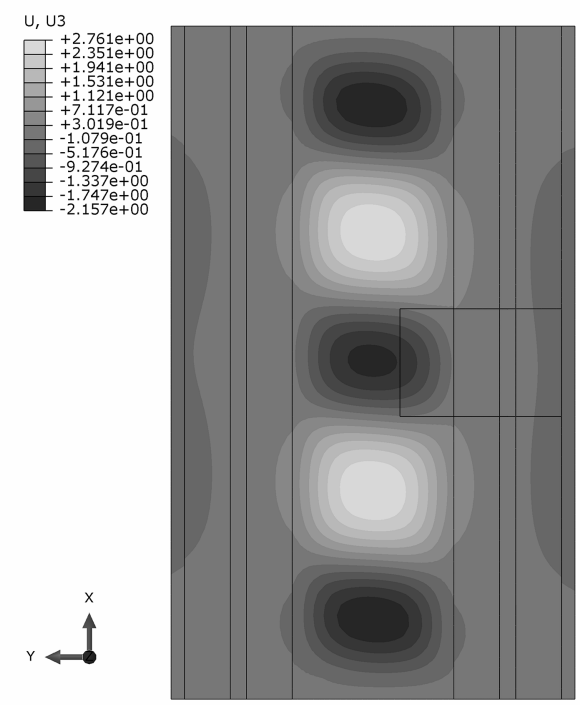

$\mathrm{F}=244 \mathrm{kN}$

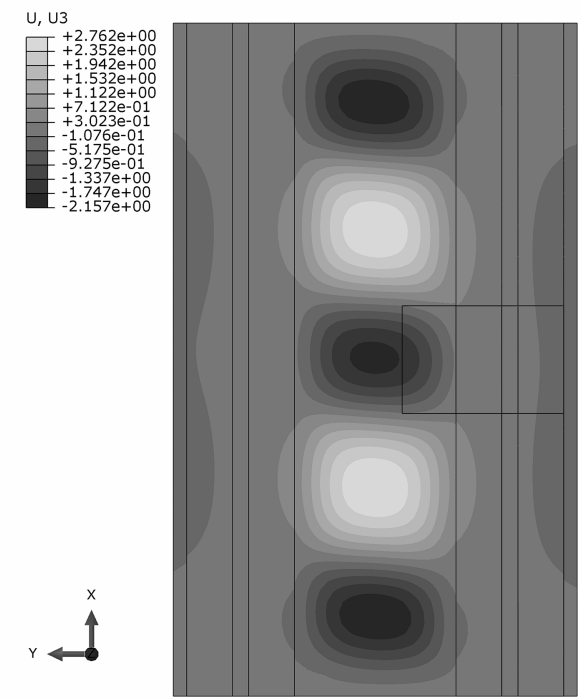

$\mathrm{F}=242 \mathrm{kN}$

Fig. 11 Comparison of the out-of-plane (U3) displacement of the sequential (left) and initialized (right) analysis, at the same load level.

ysis, into a new model. Identical results in terms of out-of-plane displacement, in-plane delaminated areas and shapes are obtained by the two simulations.

\section{Conclusions}

An original damage initialization procedure, that can function in three different modes of increasing complexity, has been developed and preliminarily assessed obtaining promising results. In this work, the procedure has been applied to composites inter-laminar damage, even though it can handle intra-laminar damage as well. Damage initialization is accomplished by means of the SDVINI subroutine that operates on an UMAT subroutine specifically developed to characterize the constitutive behavior of ABAQUS cohesive elements.

The geometric initialization mode can be easily used for analytically defined domains while, whether accurate NDI data are available, the NDI-like initialization mode is able to automatically inject the measured dam- 

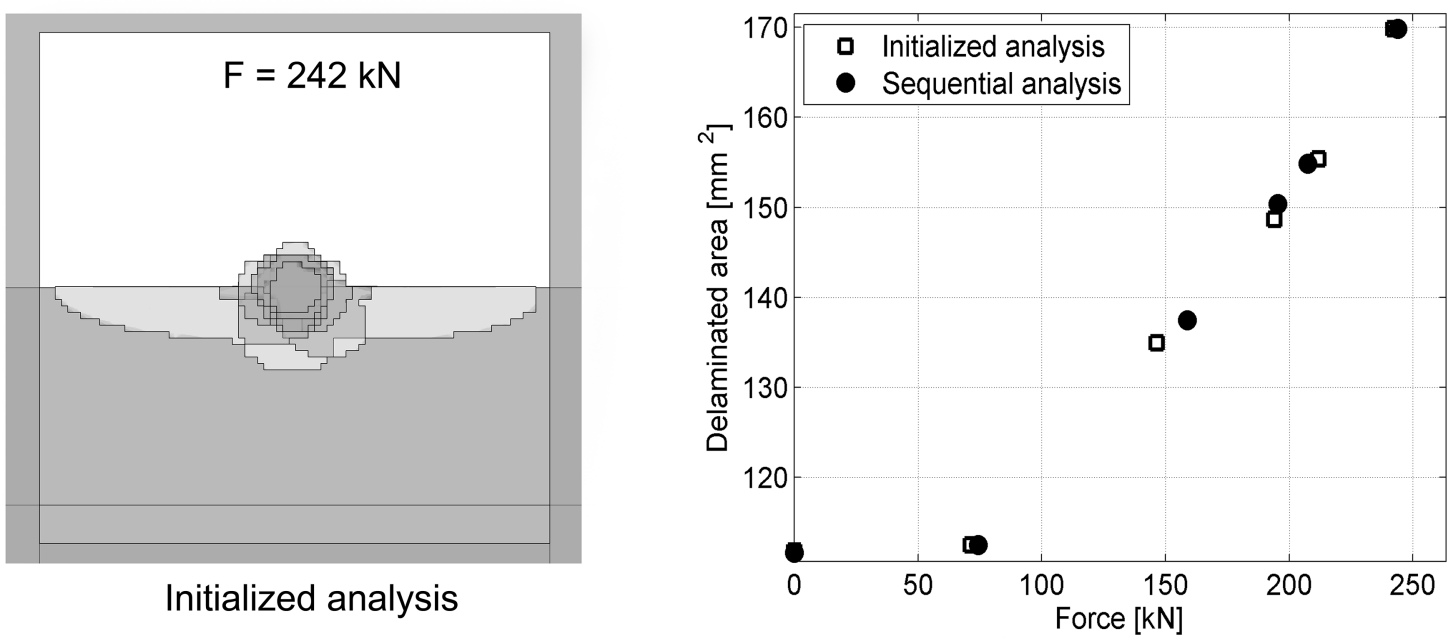

Fig. 12 Final configuration of the delaminations of the initialized analysis (left) and delaminated areas vs. applied force (right).

aged areas in the FE model of interest. Eventually, the most complex of initialization techniques developed (simulation-based mode) uses Python scripts to extract, organize and supply to the SDVINI the damage variables to be initialized and their relevant values.

Each damage initialization mode can be used to reproduce different damage scenarios on larger structures avoiding multiple sequential simulations. In particular, the simulation-based initialization technique can be used to store the results of complex damage events on a structure (such as impact events), in terms of damage variables and their location. Then, by initializing suitable larger structural models, the effects of different damage scenarios can be evaluated both on the response of the structure to damage growth and on its ultimate load carrying capability.

Eventually, for complex structures, the capability to inject damage would offer the possibility to perform engineering studies on damaged structures, without the burden of repeating the impact simulation step.

\section{References}

1. U.S. Department of Defense (2002) Composite materials handbook, vol. 1-4 F. MIL-HDBK-17 rev F.

2. Davies G, Ankersen J (2008) Virtual testing of realistic aerospace composite structures. J Mater Sci 43:65866592. doi: $10.1007 / \mathrm{s} 10853-008-2695-\mathrm{x}$

3. Donadon MV, Iannucci L, Falzon BG, Hodgkinson JM , De Almeida SFM (2008) A progressive failure model for composite laminates subjected to low velocity impact damage. Comput Struct 86:12321252.

4. Tita V, de Carvalho J, Vandepitte D (2008) Failure analysis of low velocity impact on thin composite laminates: Experimental and numerical approaches. Compos Struct 83(4):413-428.
5. Lopes CS , Camanho PP , Grdal Z, Maim P, Gonzlez EV (2009) Low-velocity impact damage on dispersed stacking sequence laminates. Part II: Numerical simulations. Compos Sci Technol 69:937-947.

6. Faggiani A, Falzon BG (2010) Predicting low-velocity impact damage on a stiffened composite panel. Compos Part A Appl Sci Manuf 41:737-749.

7. Shi Y, Swait T, Soutis C (2012) Modelling damage evolution in composite laminates subjected to low velocity impact. Compos Struct 94(9):2902-2913.

8. Raimondo L, Iannucci L, Robinson P, Curtis PT (2012) A progressive failure model for mesh-size-independent FE analysis of composite laminates subject to low-velocity impact damage. Compos Sci Technol 72(5):624-632.

9. Feng D, Aymerich F (2014) Finite element modelling of damage induced by low-velocity impact on composite laminates. Compos Struct 108:161171. doi: 10.1016/j.compstruct.2013.09.004

10. Fanteria D, Longo G, Panettieri E (2014) A non-linear shear damage model to reproduce permanent indentation caused by impacts in composite laminates. Compos Struct 111:111-121.

11. Camanho PP, Davila CG, de Moura MF (2003) Numerical simulation of mixed-mode progressive delamination in composite materials. J Compos Mater 37:14151438. doi: 10.1177/002199803034505

12. Turon A, Camanho PP, Costa J, Dvila CG (2006) A damage model for the simulation of delamination in advanced composites under variable-mode loading. Mech Mater 38:10721089. doi: 10.1016/j.mechmat.2005.10.003

13. Turon A, Dvila CG, Camanho PP, et al. (2007) An engineering solution for mesh size effects in the simulation of delamination using cohesive zone models. Eng Fract Mech 74:16651682. doi: 10.1016/j.engfracmech.2006.08.025

14. Dvila C, Camanho P, Turon A (2008) Effective simulation of delamination in aeronautical structures using shells and cohesive elements. J Aircr. doi: 10.2514/1.32832

15. Turon A, Camanho P, Costa J, Renart J (2010) Accurate simulation of delamination growth under mixed-mode loading using cohesive elements: definition of interlaminar strengths and elastic stiffness. Compos Struct 92:18571864. doi: 10.1016/j.compstruct.2010.01.012 
16. Wisnom MR (2010) Modelling discrete failures in composites with interface elements. Compos Part A Appl Sci Manuf 41:795805. doi: 10.1016/j.compositesa.2010.02.011

17. Longo G (2011) Models and methods to simulate lowenergy impact damage on composite aerospace structures. Ph.D. Thesis, University of Pisa.

18. Dassault Systemes Simulia Corp. (2014), Abaqus 6.13 User's Manual.

19. Harper PW, Sun L, Hallett SR (2012) A study on the influence of cohesive zone interface element strength parameters on mixed mode behaviour. Compos Part A Appl Sci Manuf 43:722734. doi: 10.1016/j.compositesa.2011.12.016

20. Song K, Davila C, Rose C (2008) Guidelines and parameter selection for the simulation of progressive delamination. ABAQUS Users Conf Newport, 115.

21. Reeder JR, Chunchu PB, Song K, Ambur DR (2002) Postbuckling and growth of delaminations in composite plates subjected to axial compression, 43rd AIAA/ASME/ASCE/AHS/ASC Structures, Structural Dynamics, and Materials Conference.

22. De Freitas M, Reis L (1998) Failure mechanisms on composite specimens subjected to compression after impact. Compos Struct 42:365-373.

23. Dugdale DS (1960) Yielding of steel sheets containing slits. J Mech Phys Solids 8:100104

24. Barenblatt G (1962) The mathematical theory of equilibrium cracks in brittle fracture. Adv Appl Mech 7:55129

25. Schellekens JCJ, de Borst R (1992) On the numerical integration of interface elements. Int J Numer Methods Eng 36:4366.

26. Benzeggagh ML, Kenane M (1996) Measurement of mixed-mode delamination fracture toughness of unidirectional glass/epoxy composites with mixed-mode bending apparatus. Compos Sci Technol 49:43949

27. American Society for Testing and Materials Standard Test Method for Mode I Interlaminar Fracture Toughness of Unidirectional Fiber-Reinforced Polymer Matrix Composites. ASTM D5528-01.

28. American Society for Testing and Materials Standard Test Method for Mixed Mode I - Mode II Interlaminar Fracture Toughness of Unidirectional Fiber-Reinforced Polymer Matrix Composites. ASTM D6671/D6671M-06. 